\title{
¿Es el nuevo sistema de evaluación del EEES realmente diferente del sistema tradicional?: Un análisis empírico del rendimiento académico en una asignatura de contabilidad
}

\author{
Are EHEA assessment systems really different from traditional ones? \\ An empirical analysis of academic performance in an accounting \\ subject
}

Pablo Durán Santomil (pablo.duran@usc.es)

José Manuel Maside Sanfíz (josemanuel.maside@usc.es)

Sara Cantorna Agra (sara.cantorna@usc.es)

David Rodeiro Pazos (david.rodeiro@usc.es)

Universidad de Santiago de Compostela (España)

http://dx.doi.org/10.12795/EDUCADE.2013.104.06

\begin{abstract}
RESUMEN: Este trabajo analiza los determinantes del rendimiento académico, medidos como la probabilidad de aprobar la asignatura o el examen final, a partir de las puntuaciones obtenidas en las distintas actividades de evaluación continua. Para ello se emplean los datos de una asignatura de contabilidad financiera y se cuantifican los factores determinantes del rendimiento del alumnado mediante modelos logit y probit. El ánimo es ver si el nuevo sistema de evaluación impuesto en el EEES aumenta el rendimiento del alumnado.

Los resultados revelan que, en general, las notas de las actividades de evaluación continua son determinantes estadísticamente significativos del rendimiento, si bien su impacto varía en función del criterio analizado.
\end{abstract}

PALABRAS CLAVE: Rendimiento académico, Evaluación continua, Probit, Logit, EEES.

ABSTRACT: The objective of this research is to explore the determinants of academic performance, measured as the probability of passing the course or the final exam, from the grades on the various ongoing evaluation activities. Specifically, this research uses data from a financial accounting course to identify and quantify determinants of student performance through logit and probit models. The focus is to see if the new evaluation system (European Higher Education Area, EHEA) increases student performance.

The results show that, in general, the grades of continuous activities are statistically significant determinants of performance, although its impact varies depending on the criteria analyzed.

KEYWORDS: Academic performance, Ongoing evaluation, Probit, Logit, EHEA.

Artículo de Investigación. Recibido: 14-03-13 - Versión revisada: 03-06-13, 18-07-13, Aceptado: 05-09-13

Licencia Creative Commons BY NC ND · 2013 - Asociación Española de Contabilidad y Administración de Empresas - AECA 


\section{INTRODUCCIÓN}

La Facultad de Ciencias Económicas y Empresariales de la Universidad de Santiago de Compostela (USC) ha implantado durante el curso 2009-2010 el Grado en Administración y Dirección de Empresas (ADE), ajustándose a los nuevos criterios recogidos en el marco del Espacio Europeo de Educación Superior (EEES). Esta implantación ha supuesto introducir innovaciones educativas fundamentadas en un cambio a una metodología de enseñanza activa y a un nuevo sistema de evaluación.

Mediante esta metodología debe conseguirse que el alumnado obtenga las competencias y habilidades que la sociedad demanda y que han sido establecidas en la implantación de los títulos. El sistema de evaluación del proceso de aprendizaje tradicional, basado únicamente en la calificación del examen final, debe modificarse de manera que se evalúen todas las actividades propuestas por el profesor a lo largo del curso. En este contexto, los profesores de la asignatura Contabilidad Financiera I han aplicado una metodología de enseñanza utilizando diferentes herramientas y un método de evaluación acumulativa por actividades desarrolladas por los alumnos. Ésta consiste en calificar cada una de las actividades realizadas a lo largo del cuatrimestre (participación activa en el aula y entrega individual de ejercicios, realización de trabajos en grupo, pruebas parciales y examen final).

El primer objetivo de este trabajo es analizar los factores determinantes de que los alumnos de la asignatura Contabilidad Financiera I de $2^{\circ}$ curso de Grado en ADE de la USC superen o no el examen final, en base a las puntuaciones alcanzadas en el resto de actividades de evaluación continua en el curso 2011/12 aplicando modelos logit y probit binomiales. El segundo objetivo es analizarla influencia de dichas actividades para aprobar la asignatura por medio del mismo tipo de modelos. Finalmente, deseamos conocer si el proceso de enseñanza-aprendizaje genera el desarrollo de competencias deseadas en el alumnado.

El trabajo se estructura del siguiente modo. En el segundo apartado se realiza una revisión bibliográfica de los factores determinantes del rendimiento académico del alumnado y de la importancia que tiene la formación en competencias en el nuevo marco universitario. En la sección tercera se explica la metodología de enseñanza aplicada en la asignatura. En la sección cuarta se exponen los modelos aplicados para contrastarlas hipótesis así como los resultados empíricos obtenidos. Finalmente, se presentan las principales conclusiones.

\section{REVISIÓN BIBLIOGRÁFICA E HIPÓTEIS}

\subsection{APRENDIZAJE BASADO EN COMPETENCIAS}

El Espacio Europeo de Educación Superior (EEES) surge como consecuencia del compromiso de los distintos Estados de la Unión Europea de potenciar una Europa del conocimiento, consolidando el proceso de construcción de una comunidad europea de ciudadanos. En 2001-2002, universidades de diversos países europeos se propusieron facilitar el proceso de convergencia educativa en el denominado proyecto Tuning. En dicho proyecto se incentivaba a las universidades a establecer estrategias de enseñanza y aprendizaje no sólo basadas en los contenidos sino también en las competencias. El aprendizaje de competencias pone de manifiesto la necesidad de que la Universidad se adapte a los nuevos tiempos de la "Sociedad de la Información y el Conocimiento». Este cambio implica no sólo reformular las funciones de la Universidad (Medina, 2005), requiere diversidad de métodos docentes combinados, el uso de las nuevas tecnologías, un mayor peso del trabajo autónomo de los estudiantes, cambios en el papel del profesorado, etc. 
En este contexto existe una creciente preocupación entre los docentes sobre las metodologías, técnicas e instrumentos más efectivos en la evaluación por competencias. López-Pastor (2010) señala que "una de las cosas más positivas de todo este proceso de cambio puede ser la superación del examen final como único instrumento de evaluación y calificación en la docencia universitaria", aspecto que consideramos fundamental ya que como señala Frederiksen (1984) la evaluación es el elemento principal en el éxito del proceso de enseñanza y aprendizaje, ya que aquello que se evalúa influye notablemente en lo que es aprendido (Alderson y Wall, 1993). Siguiendo a Villardón y Yániz (2004) la educación superior conlleva que la planificación y desarrollo del proceso formativo debe estar supeditado a una serie de condicionantes:

- El alumnado debe ser activo y autónomo en la construcción del propio conocimiento.

- Los aprendizajes no deben ser solo conceptuales, sino procedimentales y actitudinales.

- El alumnado debe desarrollar las competencias delimitadas según el perfil académico-profesional.

- Se deben promover situaciones de aprendizaje en condiciones "similares" al contexto de desarrollo profesional real.

Los autores de este trabajo no sólo nos planteamos conocer si los cambios establecidos en el proceso de enseñanza-aprendizaje genera el desarrollo de competencias en el alumnado sino si provoca diferencias en su rendimiento académico.

\subsection{RENDIMIENTO ACADÉMICO}

Siguiendo a Tejedor (2003) la definición de urendimiento académico» se puede establecer a través de los siguientes criterios:

- Rendimiento inmediato: Resultados y calificaciones que obtienen los alumnos a lo largo de sus estudios hasta obtener la titulación correspondiente. Se establecen tres categorías:

- Rendimiento en sentido amplio: Éxito (finalización puntual de una titulación en los años previstos en el plan de estudios); retraso (finalización empleando más tiempo del establecido oficialmente) y abandono de estudios.

- Regularidad académica: Tasas de presentación o no a los exámenes.

- Rendimiento en sentido estricto: Notas obtenidas por los estudiantes.

- Rendimiento diferido: Se refieren a la aplicación o utilidad que la formación recibida tiene en la vida laboral y social, cuya valoración es mucho más compleja.

Los factores que influyen en el rendimiento académico de los alumnos son de naturaleza diversa por lo que son difíciles de identificar. Tejedor (2003) establece cinco categorías de variables para el análisis:

- Variables de identificación (género, edad).

- Variables psicológicas (aptitudes intelectuales, personalidad, motivación, hábitos de estudios, etc.) 
- Variables académicas (tipos de estudios cursados, curso, opción en que se estudia una carrera, rendimiento previo, asistencia a clase, etc.)

- Variables pedagógicas (método de enseñanza, estrategias de evaluación, etc.).

- Variables socio-familiares (estudios de los padres, situación laboral de los padres, etc.)

En muchos de los numerosos estudios sobre los denominados «determinantes del rendimiento académicol se obtiene que los mejores predictores del rendimiento académico son las variables académicas, en especial el rendimiento académico previo. En la literatura especializada las variables académicas que determinan el rendimiento han sido tradicionalmente la nota de acceso a la universidad, las notas de otras asignaturas de cursos pasados, las notas de asignaturas del mismo departamento, etc. Nuestro trabajo analizará modelos de regresión de las variables que determinan el rendimiento inmediato en sentido estricto (aprobar el examen final y aprobar la asignatura) en función de las notas alcanzadas en el proceso de evaluación continua por actividades, por lo que a continuación nos centraremos en la revisión de los trabajos que analizaron en alguna medida estas variables.

\subsubsection{ASISTENCIA}

En primer lugar, son múltiples las investigaciones que presentan asociaciones positivas entre asistencia y rendimiento, si bien en algunos trabajos ésta no aparece como variable esencial. Esto puede ser debido a que a ciertos alumnos les sea más rentable dedicar el tiempo invertido en las clases al estudio individual o las dificultades que requiere su presencia, dadas las horas trabajadas y el tiempo del viaje a la universidad. En la literatura especializada se acepta que los alumnos más capaces, motivados y trabajadores son más propensos tanto a asistir como a obtener altas calificaciones. La asistencia refleja una mayor participación en clase y es una medida de la motivación del alumno, además tiene externalidades positivas para otros estudiantes a través de las contribuciones que cada uno puede aportar al proceso de aprendizaje (Arulampalam et al. 2012). Romer (1993) encuentra que el efecto de la asistencia a clase es siempre positivo y significativo, sin embargo, su magnitud se reduce enormemente por la inclusión de proxies de la motivación en las regresiones sobre el rendimiento académico. Durden y Ellis (1995) encuentran un efecto no lineal en la relación: mientras un bajo nivel de ausencias no conducen a peores calificaciones, un ausentismo elevado si está asociado. Devadoss y Foltz (1996) encuentran un efecto positivo significativo entre la asistencia y el desempeño incluso después de tener en cuenta las diferencias de motivación y aptitud a través de los estudiantes. Rodgers (2002) implementó un plan de incentivos para la asistencia a clase por parte de los alumnos, obteniendo que la mejora de la asistencia no se tradujera en un mejor rendimiento académico. Sin embargo, dentro de cada curso, encuentra una relación fuerte entre asistencia y desempeño. Stanca (2006) concluye que la asistencia tiene un importante efecto sobre el rendimiento académico. Delaney et al. (2011) encuentran que la asistencia a clase es un factor predominante a la hora de explicar el rendimiento académico. Arulampalam et al. (2012) encuentran que los alumnos que no asisten a clase obtienen peores resultados. Sin embargo, Martins y Walker (2006) no encuentran efectos positivos sobre la asistencia a clase y el rendimiento obtenido por el alumno, ni que en las clases con menor número de alumnos se obtengan mejores resultados.

En este punto esperamos que la nota obtenida en la participación activa en clase y la realización de trabajos individuales (variable participación) tenga una influencia positiva sobre la probabilidad de aprobar el examen final y la probabilidad de aprobar 
la asignatura. Para alcanzar una alta puntuación en esta variable el alumno debe tener un grado de asistencia elevado a clase, participar activa y adecuadamente en la resolución de ejercicios en el aula y además entregar correctamente resueltos los trabajos que se proponen y recogen en la misma.

H1: Los alumnos que obtienen una mayor nota en "participación" tienen una mayor probabilidad de aprobar el examen final.

H2: Los alumnos que obtienen una mayor nota en "participación" tienen una mayor probabilidad de aprobar la asignatura.

\subsubsection{PRUEBAS PARCIALES}

Trabajos como el de Rúa y González (2004) tratan de predecir el rendimiento académico a partir de pruebas previas, encontrando que todos los controles influyen positivamente, es decir, cuanto mejor nota se obtiene en las pruebas mejor es el rendimiento académico y mayor es la probabilidad de aprobar la asignatura. Por su parte, Barkley (2006) analiza la influencia del método de evaluación sobre el rendimiento de los estudiantes. Encuentra que las correlaciones alcanzadas por los alumnos en base a un sistema de evaluación formado por tareas, cuestionarios, exámenes parciales y un examen final no son muy elevadas, con unos coeficientes de correlación de Pearson que van de 0,40 a 0,72 , aunque en todos los casos son significativos al $1 \%$. Los exámenes parciales fueron los más estrechamente relacionados con la nota final del curso $(0,94)$. De las regresiones efectuadas, se encuentra que las notas del examen final parecen no estar influenciadas por las calificaciones en las tareas o en los cuestionarios, pero sí por las de los exámenes parciales. Los coeficientes estimados muestran que los resultados del estudiante varían ampliamente entre los tipos de evaluación, incluso para los mismos estudiantes. Estos cambios pueden ser debidos a diferencias en el estilo de aprendizaje, la capacidad o la experiencia del alumno con diferentes métodos de evaluación. Pascual et al. (2011) muestran que la evaluación acumulativa por actividades en el nuevo marco del EEES puede presentar limitaciones, en la medida en que los alumnos que sacan mejores notas en el examen final ven reducida su calificación final como resultado de las actividades de evaluación continua.

En este punto esperamos que las notas alcanzadas en las pruebas parciales tengan una influencia positiva en la nota alcanzada en el examen final y en la asignatura, ya que implican un seguimiento continuo en el estudio de la asignatura y su correcta comprensión. Además, los ejercicios presentados en el examen final son muy similares a los realizados en las pruebas de evaluación continua, por lo que se espera que nuevamente tenga una influencia positiva sobre la probabilidad de aprobar el examen final y la asignatura. Dado que el temario de la asignatura abordado es extenso y los distintos temas están fuertemente relacionados conceptualmente, se espera que los alumnos que alcanzan buena nota en una prueba la alcancen en el resto, y por supuesto, en el examen final y en la asignatura. Las ventajas que pueden tener las pruebas parciales para mejorar el proceso de aprendizaje del estudiante son múltiples. En primer lugar, las pruebas proporcionan información valiosa para los estudiantes y los profesores. Los estudiantes obtienen un feedback de lo bien o mal que las están haciendo, y si es necesario un cambio en los niveles de esfuerzo o si necesitan recibir ayuda o apoyo (tutorías por parte del profesor o del exterior). También proporcionan información a los profesores sobre el grado en que los estudiantes están aprendiendo, y si es necesario ajustes en el contenido, el ritmo, etc. Es decir, pueden identificarse dificultades específicas tanto por los estudiantes como por los profesores y realizar los ajustes pertinentes antes del examen final. 
H3: Los alumnos que obtienen una mayor nota en cada una de las pruebas parciales realizadas ("prueba 1", "prueba 2", "prueba 3") tienen una mayor probabilidad de aprobar el examen final.

H4: Los alumnos que obtienen una mayor nota en cada una de las pruebas parciales realizadas ("prueba 1", "prueba 2", "prueba 3") tienen una mayor probabilidad de aprobar la asignatura.

\subsubsection{TRABAJO EN EQUIPO}

La realización del trabajo en grupo debiera tener influencia positiva en la medida en que su elaboración refleja un interés por la asignatura, sirve para ver su aplicación práctica, requiere una mínima comprensión de los conceptos adquiridos y enlaza todos los temas tratados en la misma. Por ello cabe esperar que los alumnos que alcancen una mayor nota en el trabajo en grupo tengan una mayor probabilidad de aprobar tanto el examen final como la asignatura. El trabajo en equipo es una técnica didáctica que capacita para realizar actividades en común aprovechando el potencial de las relaciones interpersonales. Se emplearon grupos reducidos formales de estudiantes que trabajaron juntos durante todo el cuatrimestre para maximizar su propio aprendizaje y el de los demás. La organización interna del grupo era responsabilidad de los alumnos, mientras que el profesor actuaba de guía o tutor, facilitando el proceso de aprendizaje.Nuestras hipótesis de partida al respecto son:

H5: Los alumnos que obtienen una mayor nota la realización del trabajo en grupo ("trabajo") tienen una mayor probabilidad de aprobar el examen final.

H6: Los alumnos que obtienen una mayor nota la realización del trabajo en grupo ("trabajo") tienen una mayor probabilidad de aprobar la asignatura.

\section{METOdOLOGÍA DE ENSEÑANZA-EVALUACIÓN}

La presente investigación se llevó a cabo con estudiantes de segundo curso de la asignatura "Contabilidad Financiera I", en el grado en ADE durante el curso académico 2011/2012. Se trata de una asignatura obligatoria cuatrimestral de 6 créditos ECTS, que se imparte en el segundo cuatrimestre. Las clases fueron impartidas en cuatro grupos expositivos de estudiantes y diez interactivos. El tiempo dedicado a la docencia en esta asignatura es de cincuenta horas en el cuatrimestre, de las cuales diecisiete se imparten a cada grupo expositivo completo y treinta y una en forma de seminarios, en las que se divide al grupo en diversos subgrupos para permitir unas clases más personalizadas y dinámicas '. Las clases expositivas consisten en la discusión de los conceptos teóricos de las transparencias de cada tema previamente publicados en la plataforma Blackboard (aula virtual) y en formato papel. Esta formación se complementa en las clases de seminario con la realización de supuestos prácticos donde los alumnos deben participar activamente resolviéndolos colectivamente en el aula. Adicionalmente deberán resolver supuestos prácticos similares individualmente dentro del aula y entregarlos al profesor. Estas actividades se evalúan en el aparado denominado "Participación activa en clase y entrega de ejercicios", cuya nota posteriormente será referida como la variable participación.

Los alumnos deberán realizar en grupos, de un máximo de tres personas, un trabajo que consiste en resolver diversas cuestiones relacionadas con la problemática contable abordada en el aula. Las preguntas formuladas versan sobre diversos aspectos de las cuentas anuales de una empresa del mercado continuo de la bolsa española asignada aleatoriamente por los profesores. Los alumnos deben buscar las

\footnotetext{
${ }^{1}$ Las otras dos horas se corresponden con tutorías interactivas.
} 
cuentas de la empresa y año que se le asigna en la web de la CNMV y contestar a cada una de las cuestiones planteadas. Las preguntas de dicho trabajo han sido separadas en tres bloques, en función de los distintos temas del programa de la asignatura, y se establecía una fecha máxima para la entrega de cada parte. Esta actividad se evalúa en el apartado denominado "Trabajos en grupo", a la nota alcanzada por los alumnos nos referiremos posteriormente como la variable trabajos.

A lo largo del cuatrimestre el alumno puede realizar tres pruebas parciales no eliminatorias en las que podrá obtener un feedback de los conocimientos adquiridos hasta esa fecha. Esta actividad se evalúa en el apartado denominado "Pruebas parciales". El carácter de estas pruebas era fundamentalmente de carácter práctico y se requería que el alumno alcanzase un mínimo de 3,5 puntos sobre 10 para su cómputo en la nota final. En última instancia, se realiza un examen final formado por múltiples preguntas tipo test y varios ejercicios prácticos valorándose en el apartado "Examen final". Cada uno de los profesores ha aplicado la misma metodología de docencia y el mismo temario, material en las clases expositivas y de seminario y sistema de evaluación. El apartado "Trabajos en grupo", "Pruebas parcial" y "Examen final" ha sido calificado para todos los alumnos por el mismo profesor independientemente del grupo al que perteneciesen, mientras que para la valoración de la "Participación activa en clase y entrega de ejercicios" se han establecido unos ítems objetivos para la valoración de cada profesor $^{2}$. El peso asignado a cada apartado del sistema de evaluación acumulativa por actividades ha sido conforme a la horquilla fijada en la Memoria del título de Grado.

\section{Gráfico 1.- Sistema de eVAluación acumulativa}

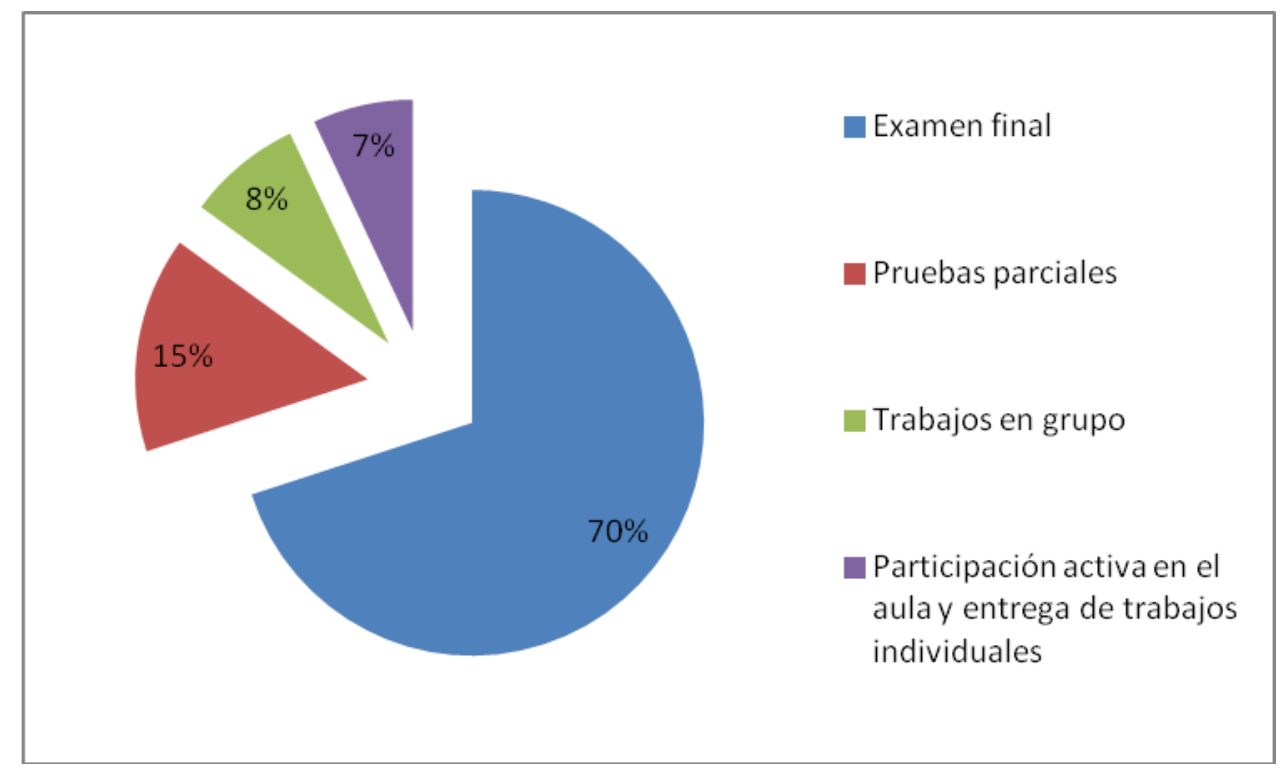

En el sistema de evaluación acumulativa (Gráfico 1), la nota del examen final supone el $70 \%$ de la nota total de la asignatura. El contenido del examen final incluyó toda la materia impartida en clase. Para realizar el examen final no era imprescindible que los estudiantes hubieran realizado ninguna de las actividades propuestas en la evaluación continua, si bien era aconsejable. Además, ningún alumno podía superar la asignatura sin alcanzar un mínimo del $40 \%$ de la puntuación del examen final en cada una de las partes (test y ejercicios). El otro $30 \%$ de la nota total se compone de la calificación de

\footnotetext{
2 Se ha establecido las valoraciones en función de los días de participación y corrección del alumno en clase y la entrega y exactitud de los trabajos individuales realizados.
} 
las distintas actividades que se desarrollaron: pruebas parciales, trabajos realizados en grupo y la participación activa y trabajos entregados en clase. Se realizaron tres exámenes parciales (cada uno con una ponderación del 5\%), que se llevaron a cabo aproximadamente una vez impartida cada tercio de la materia (temas 1 a 4 , temas 5 a 8 , temas 9 a 11), para comprobar los conocimientos adquiridos por parte de los estudiantes. El trabajo en grupo representaba el $8 \%$ sobre la nota total. El restante $7 \%$ de la nota total se basa en la participación activa del estudiante en clase y la realización, entrega y posterior corrección de ejercicios cortos. El sistema de evaluación era independiente de la condición de repetidor o no y de la fecha de presentación a examen (junio o julio).

Dado que el aprendizaje por parte de los alumnos requiere la diversidad de métodos docentes combinados se han facilitado los materiales y recursos que faciliten el aprendizaje autónomo, mediante la elaboración por los profesores de la asignatura de un libro de ejercicios y de supuestos resueltos y la incorporación en el aula virtual de diferentes artículos de interés, supuestos resueltos y sin resolver, noticias y enlaces de interés y test de autoevaluación mediante preguntas tipo test. La planificación docente se ha materializado a través de una guía docente difundida en la plataforma virtual. Finalmente también se han desarrollado prácticas informáticas como metodologías docentes que nos parecían adecuadas para la adquisición de competencias.

\section{MODELOS Y RESULTADOS ALCANZADOS}

\subsection{MUESTRA}

La muestra inicial está formada por 310 alumnos de la asignatura Contabilidad Financiera I de $2^{\circ}$ curso de Grado en ADE de la USC en el curso 2011/12 que han participado en alguna de las actividades de evaluación desarrolladas, si bien el número de casos válidos en cada análisis es variable. De esta forma, la muestra no es uniforme en todas las fases del ensayo debido a que no todos los alumnos han realizado todas las actividades de evaluación propuestas o no han contestado a los cuestionarios realizados. En este sentido, en el análisis posterior de diferencias de medias se observa un abandono del número de alumnos después de la primera prueba cuando la calificación obtenida es excesivamente baja. En el análisis de los coeficientes de regresión entre las variables y los modelos econométricos logit y probit se ha realizado únicamente con los 149 alumnos que han realizado todas las actividades de evaluación continua.Para los cuestionarios sobre las competencias alcanzadas en el trabajo en equipo se emplearon los datos de los 250 alumnos que contestaron al mismo.

\subsection{ANÁLISIS ESTADÍSTICO PREVIO}

Con el objeto de analizar si los alumnos que realizan las actividades de evaluación continua tienen una mayor probabilidad de superar el examen final se han calculado en primer lugar las diferencias de medias entre los alumnos que superan y no superan dicho examen (variable dicotómica que toma el valor 0 cuando el alumno obtiene una puntuación inferior a 5 y toma el valor 1 cuando se obtiene una valor igual 0 superior a 5). Así, los alumnos que superan el examen final (66 en total) tienen en promedio una mayor nota en cada una de las tres pruebas parciales, en la participación activa en clase y en el trabajo en grupo, siendo dichas diferencias significativas al $1 \%$.

En concreto, los alumnos que aprueban el examen final tienen una media de 7,33 puntos en la primera prueba frente a los 3,95 puntos alcanzados por los alumnos que 
no aprueban dicho examen. La nota en la segunda prueba es mayor en aquellos alumnos que superan el examen $(7,62)$ frente a las que no lo superan $(3,04)$. De nuevo, la nota en la tercera y última prueba es mayor en aquellos alumnos que superan el examen $(8,30)$ frente a las que no lo superan $(4,30)$. La nota media ponderada de las pruebas, eliminando las calificaciones de los alumnos inferiores a 3,5 puntos, obtenida por los alumnos que aprueban el examen final es de 7,45 puntos frente a los 2,24 puntos alcanzados por los alumnos que no aprueban el examen. Fruto de los anteriores resultados, podemos concluir que el estudio continuo de la asignatura a lo largo del cuatrimestre parece influir positivamente sobre la nota del examen final.

La nota en el trabajo en grupo es mayor en aquellos alumnos que superan el examen $(9,72)$ frente a las que no lo superan $(8,97)$. Por último, la nota alcanzada en la participación en clase (participación activa en la resolución de ejercicios y entrega de supuestos) es nuevamente superior en los alumnos que superan el examen $(7,64)$ frente a las que no lo superan $(5,34)$. Además del estudio continuo de la asignatura a lo largo del cuatrimestre, semeja que la participación activa en el aula y la realización de trabajos (individuales y en grupo) influye positivamente sobre la probabilidad de aprobar el examen final, ya que son variables que miden indirectamente el seguimiento continuo de la asignatura y el interés en la misma.

Por otra parte, se han analizado las correlaciones de Pearson existentes entre la variable dicotómica aprobar el exámenes final ( 0 en caso negativo y 1 en caso afirmativo) y las de las distintas pruebas realizadas, participación y realización del

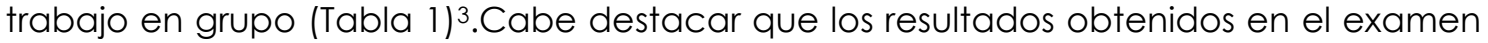
final correlacionan de forma positiva y significativa al $1 \%$ con las tres pruebas parciales, la nota ponderada de las pruebas y la participación activa en clase. La correlación entre la variable dicotómica aprueba el examen final y la nota alcanzada en los trabajos en grupo es la de menor coeficiente de correlación lineal, no resultando significativa al $1 \%$ pero sí al $2,5 \%$.

TABLA 1.Correlación lineal entre las Variables

\begin{tabular}{|c|c|c|c|c|c|c|c|}
\hline & $\begin{array}{l}\text { Aprueba } \\
\text { examen } \\
\text { final }\end{array}$ & $\begin{array}{l}\text { Prueba } \\
\quad 1\end{array}$ & $\begin{array}{l}\text { Prueba } \\
\quad 2\end{array}$ & $\begin{array}{c}\text { Prueba } \\
\quad 3\end{array}$ & $\begin{array}{c}\text { Nota } \\
\text { ponderada } \\
\text { pruebas }\end{array}$ & Trabajo & Participación \\
\hline $\begin{array}{c}\text { Aprueba } \\
\text { examen final }\end{array}$ & 1,000 & & & & & & \\
\hline Prueba 1 & $\begin{array}{c}0,598 \\
(0,000)\end{array}$ & 1,000 & & & & & \\
\hline Prueba 2 & $\begin{array}{c}0,669 \\
(0,000)\end{array}$ & $\begin{array}{c}0,578 \\
(0,000)\end{array}$ & 1,000 & & & & \\
\hline Prueba 3 & $\begin{array}{c}0,562 \\
(0,000)\end{array}$ & $\begin{array}{c}0,501 \\
(0,000)\end{array}$ & $\begin{array}{c}0,609 \\
(0,000)\end{array}$ & 1,000 & & & \\
\hline $\begin{array}{c}\text { Nota } \\
\text { ponderada } \\
\text { pruebas }\end{array}$ & $\begin{array}{c}0,705 \\
(0,000)\end{array}$ & $\begin{array}{c}0,782 \\
(0,000)\end{array}$ & $\begin{array}{c}0,867 \\
(0,000)\end{array}$ & $\begin{array}{c}0,838 \\
(0,000)\end{array}$ & 1,000 & & \\
\hline Trabajos & $\begin{array}{c}0,196 \\
(0,017)\end{array}$ & $\begin{array}{c}0,293 \\
(0,000)\end{array}$ & $\begin{array}{c}0,253 \\
(0,002)\end{array}$ & $\begin{array}{c}0,261 \\
(0,001)\end{array}$ & $\begin{array}{c}0,347 \\
(0,000)\end{array}$ & 1,000 & \\
\hline Participación & $\begin{array}{c}0,438 \\
(0,000)\end{array}$ & $\begin{array}{c}0,478 \\
(0,000)\end{array}$ & $\begin{array}{c}0,404 \\
(0,000)\end{array}$ & $\begin{array}{c}0,390 \\
(0,000)\end{array}$ & $\begin{array}{c}0,512 \\
(0,000)\end{array}$ & $\begin{array}{c}0,261 \\
(0,001)\end{array}$ & 1,000 \\
\hline
\end{tabular}

Nota: Se muestran los coeficientes de correlación lineal entre las distintas variables y el p-valor asociado a su significatividad entre paréntesis.

\footnotetext{
3 Se han analizado también el coeficiente de correlación de Spearman y la tau de Kendall obteniendo resultados muy similares tanto en coeficientes como en significatividad.
} 


\subsection{ANÁLISIS EMPÍRICO}

Los modelos probit y logit pueden ser empleados para analizar la probabilidad de superar el examen final. Estos modelos establecen una relación no lineal entre una variable endógena que toma únicamente dos valores $\left(y_{i}=1\right.$, cuando el alumno aprueba el examen final e $y_{i}=0$, cuando no aprueba) y un conjunto $K$ de variables independientes. De esta forma, en un modelo de regresión para $i=1, \ldots, N$ :

$$
y_{i}=\beta_{1} x_{1 i}+\ldots+\beta_{K} x_{K i}+\varepsilon_{i}
$$

Donde $\beta_{i}$ son los coeficientes de las variables explicativas y $\varepsilon_{i}$ es el término de perturbación con media cero. El valor esperado del modelo puede interpretarse como la probabilidad de que el alumno apruebe el examen. Para obtener un modelo que reproduzca adecuadamente el comportamiento de una función de probabilidad es frecuente emplear la distribución normal estándar (modelo probit) o la logística (modelo logit). Los coeficientes o parámetros $\beta$ de un modelo probit o logit no son fáciles de interpretar directamente, siendo común emplear los efectos marginales.

En esta parte del trabajo analizamos, mediante modelos logit y probit, la probabilidad de aprobar el examen final en base a las puntuaciones obtenidas en el resto de actividades de evaluación continúa desarrolladas por el alumno. Dados los potenciales problemas de multicolinealidad que podrían resultar de la consideración conjunta de las variables pruebas 1, 2 y 3 con la nota conjunta de las pruebas, hemos decido excluir de los posteriores análisis esta última ${ }^{4}$. La Tabla 2 recoge las estimaciones probit y logit de la relación entre la probabilidad de aprobar el examen y las variables independientes mencionadas y la significatividad de los coeficientes. También se muestran los efectos marginales (E.M.) de los cambios en las variables independientes sobre el valor de la variable dependiente observada. Los efectos marginales han sido calculados para cada observación de la muestra, obteniendo los efectos medios como promedio de los efectos individuales. Los modelos 1, 2 y 3 son modelos probit mientras que los modelos 4, 5 y 6 son modelos logit. Se aprecia como las variables notas alcanzadas en las tres pruebas parciales tienen una influencia positiva sobre la probabilidad de aprobar el examen final en todos los modelos, resultando siempre variables significativas al 1\%. La variable participación no resulta significativa en los modelos que se incorpora como variable dependiente. Finalmente, y contrario a nuestra hipótesis de partida inicial, la variable trabajo en grupo tiene una influencia negativa en todos los modelos, siendo significativa en aquellos que se elimina el término de constante. Además en estos casos, el valor del efecto marginal de esta variable indica que es el factor que tiene mayor peso sobre la probabilidad de aprobar el examen. Para analizar la bondad de ajuste de los modelos se emplea el test de Hosmer y Lemeshow. De los p-valores asociados a los seis modelos analizados resulta que el test no es significativo, lo que implica un buen ajuste de los modelos. Es decir, no se rechaza la hipótesis nula de que el modelo se ajusta adecuadamente a las observaciones.

\footnotetext{
${ }^{4}$ El test de Farrar-Glauber no detecta multicolinealidad entre las variables empleadas en el modelo.
} 
Pablo Durán, José M. Maside, Sara Catorna, David Rodeiro

¿Es el nuevo sistema de evaluación del EESS realmente diferente del sistema tradicional?

TABLA 2. ESTIMACIONES DE LOS MODELOS PROBIT Y LOGIT

PARA LA PROBABILIDAD DE APROBAR EL EXAMEN FINAL

\begin{tabular}{|c|c|c|c|c|c|c|c|c|c|c|c|c|}
\hline & \multicolumn{12}{|c|}{ Variable dependiente: Aprueba el examen final $=1$, No aprueba el examen final $=0$} \\
\hline & \multicolumn{2}{|c|}{$\begin{array}{c}\text { Modelo1 } \\
\text { Probit }\end{array}$} & \multicolumn{2}{|c|}{$\begin{array}{c}\text { Modelo } 2 \\
\text { Probit }\end{array}$} & \multicolumn{2}{|c|}{$\begin{array}{c}\text { Modelo } 3 \\
\text { Probit }\end{array}$} & \multicolumn{2}{|c|}{$\begin{array}{c}\text { Modelo } 4 \\
\text { Logit }\end{array}$} & \multicolumn{2}{|c|}{$\begin{array}{c}\text { Modelo } 5 \\
\text { Logit }\end{array}$} & \multicolumn{2}{|c|}{$\begin{array}{c}\text { Modelo } 6 \\
\text { Logit }\end{array}$} \\
\hline & Coef. & E.M & Coef. & E.M & Coef. & E.M & Coef. & E.M & Coef. & E.M & Coef. & E.M \\
\hline Prueba 1 & $\begin{array}{c}0,25 \\
(* * *)\end{array}$ & 0,04 & $\begin{array}{l}0,26 \\
(* * *)\end{array}$ & 0,04 & $0,20(* * *)$ & 0,04 & $0,49(* * *)$ & 0,05 & $0,49(* * *)$ & 0,05 & $0,39(* * *)$ & 0,04 \\
\hline Prueba 2 & $\begin{array}{c}0,23 \\
(* * *)\end{array}$ & 0,04 & $0,23(* * *)$ & 0,04 & $0,21(* * *)$ & 0,04 & $0,39(* * *)$ & 0,04 & $0,39(* * *)$ & 0,04 & $0,37(* * *)$ & 0,04 \\
\hline Prueba 3 & $\begin{array}{l}0,13 \\
(* *)\end{array}$ & 0,02 & $0,14\left({ }^{* *}\right)$ & 0,02 & $0,17(* * *)$ & 0,03 & $0,25(* *)$ & 0,02 & $0,26(* * *)$ & 0,02 & $0,32(* * *)$ & 0,03 \\
\hline Trabajos & $-0,15$ & 0,02 & $\begin{array}{l}-0,48 \\
(* * *) \\
\end{array}$ & 0,08 & $0,40(* * *)$ & 0,08 & $-0,42$ & 0,09 & $0,91(* * *)$ & 0,09 & $\stackrel{-}{-}, 74(* *))$ & 0,08 \\
\hline Participación & 0,10 & 0,02 & 0,09 & 0,02 & & & 0,20 & 0,02 & 0,21 & 0,02 & & \\
\hline Constante & $-3,18$ & & & & & & $-4,59$ & & & & & \\
\hline $\begin{array}{c}\text { Log } \\
\text { likelihood }\end{array}$ & $\begin{array}{c}- \\
45,73 \\
\end{array}$ & & $-46,25$ & & $-65,89$ & & $-45,36$ & & $-45,68$ & & $-65,19$ & \\
\hline AIC & 0,69 & & 0,69 & & 0.71 & & 0,69 & & 0,68 & & 0,71 & \\
\hline$S B C$ & 0,82 & & 0,79 & & 0.78 & & 0,81 & & 0,78 & & 0,77 & \\
\hline $\mathrm{HQC}$ & 0,74 & & 0,73 & & 0.74 & & 0,74 & & 0,72 & & 0,73 & \\
\hline $\mathrm{R}^{2}$ Mcfadden & 0,53 & & & & & & 0,54 & & & & & \\
\hline $\begin{array}{c}\text { Hosmer- } \\
\text { Lemeshow } \\
X^{2}\end{array}$ & 8,70 & & 10,39 & & 9,49 & & 6,31 & & 7,97 & & 6,19 & \\
\hline
\end{tabular}

Notas: ${ }^{* * * * *}, *$ hace referencia a coeficientes significativos al 0,01, 0,05 y 0,1 respectivamente. El término Log likehood se refiere al valor logaritmo de la función de verosimilitud de cada modelo. Los criterios AIC, SBC y HQC, Akaike (1973), Schwarz (1978) y Hannan-Quinn (1979) respectivamente, sirven para comparar la bondad del ajuste entre modelos. Según estos criterios es preferible aquel modelo que presente un valor menor. El tradicional coeficiente de determinación $\mathrm{R}^{2}$ no es válido en los modelos en los que las variables endógenas toman exclusivamente los valores de uno o cero, utilizándose el $R^{2}$ propuesto por McFadden (1974). La prueba de Hosmer y Lemeshow (1989) calcula para cada observación las probabilidades de la variable dependiente que predice el modelo, a partir de ellas compara las frecuencias esperadas con las observadas. 
Los anteriores resultados nos llevan a plantearnos la utilidad de la elaboración del trabajo en grupo para los alumnos, y si la realización de dicho trabajo y de la participación activa en el aula tiene influencia sobre la capacidad de superar la asignatura, aún siendo incapaces de aprobar el examen final.

La principal ventaja de los trabajos en equipo es que no sólo se aprenden conocimientos y cómo aplicarlos, sino también numerosas habilidades como son la comunicación interpersonal, el trabajo en equipo, el liderazgo, etc. Sin embargo, cualquier trabajo en grupo puede presentar distintos inconvenientes como cualquier otra técnica docente, entre los que destacan: el bajo grado de compromiso de alguno de los componentes, elevada inversión de tiempo en el trabajo, falta organización del trabajo en equipo, etc. En Bolonia, el proceso de enseñanzaaprendizaje aboga por un nuevo estilo docente que potencie el desarrollo de competencias en el alumnado. Deseamos analizar si la realización del trabajo en grupo capacita a los alumnos para prepararse como profesional, en una sociedad que requerirá adaptarse a situaciones cambiantes y que se tengan que aplicar diversos conocimientos para solucionar problemas reales. Para ello se ha realizado una encuesta anónima a los alumnos que han realizado el trabajo en grupo. En la Tabla 3 se muestra la valoración de los mismos acerca del desarrollo de las competencias desarrolladas con la realización del trabajo. Se observa como en media todas las competencias resultan superiores a la puntuación media de la escala empleada (5). Las cinco principales competencias desarrollas en el trabajo resultan ser: trabajo en equipo, habilidad para buscar y analizar información, conocimientos generales básicos sobre contabilidad, capacidad de aplicar los conocimientos en la práctica y la capacidad de aprender. En la misma tabla se aprecian también distintas opiniones sobre la realización del trabajo. Se observan opiniones positivas respecto a que la realización de dicho trabajo permite ver la aplicación práctica de la asignatura, relacionar todos los temas de la asignatura y aprender cosas útiles, de forma que en media los alumnos afirman que les ha ayudado a entender mejor la asignatura. Sin embargo, como aspectos negativos del mismo afirman que la realización de dicho trabajo requiere más tiempo del previsto por el equipo docente por lo que el esfuerzo no se corresponde con la valoración en la nota final de la asignatura. Además, también afirman que en media no les ha servido para preparar el examen final de la asignatura. Esta afirmación parece estar en consonancia con el resultado alcanzado en los modelos probit y logit anteriores. Sin embargo, pese a los anteriores inconvenientes observados por los alumnos, éstos no preferirían realizar el trabajo de forma individual, un $71 \%$ lo volverían a realizar si tuvieran que repetirlo y un $75 \%$ se muestra globalmente satisfecho con la realización del mismo.

En segundo lugar deseamos analizar si la realización del trabajo en grupo y de la participación activa en el aula tiene influencia sobre la capacidad de superar la asignatura, a pesar de que parece no tener influencia sobre la probabilidad de aprobar el examen. Del análisis estadístico del número de alumnos que aprueban el examen (66 en total) y la asignatura (93 alumnos) observamos los siguientes hechos:

- Hay 29 alumnos que han superado la asignatura aún no siendo capaces de alcanzar una puntuación de 5 en el examen final. Todos ellos se han presentado a las tres pruebas parciales. De ellos 25 alumnos (todos excepto 4) han participado en clase, y todos ellos han elaborado el trabajo en grupo.

- Todos los alumnos que han superado el examen final menos dos han superado la asignatura, es decir, 64 alumnos han superado el examen y la asignatura. Todos los alumnos que han superado el examen final menos nueve han participado en las clases de seminario. Todos los alumnos que han superado el examen final menos dos han realizado el trabajo en grupo. Todos los alumnos 
que han superado el examen final menos tres han realizado todas las pruebas parciales.

TABLA 3: ENCUESTA DE SATISFACCIÓN Y COMPETENCIAS DESARROLLADAS EN EL TRABAJO EN GRUPO

\section{Competencias}

¿Crees que la realización del trabajo en grupo te ha permitido desarrollar/mejorar la habilidad/competencia de?:

Puntúa de 0-10 (0 muy en desacuerdo 10 totalmente de acuerdo) la habilidad/competencia desarrollada en el trabajo de esta asignatura.

\begin{tabular}{|l|c|}
\hline Trabajo en equipo & 6,76 \\
\hline Habilidad para buscar y analizar información & 6,71 \\
\hline Conocimientos generales básicos sobre contabilidad & 6,66 \\
\hline Capacidad de aplicar los conocimientos en la práctica & 6,42 \\
\hline Capacidad de aprender & 6,22 \\
\hline Habilidades interpersonales & 6,05 \\
\hline Capacidad de análisis y síntesis & 6,00 \\
\hline Resolución de problemas & 5,97 \\
\hline Habilidad para trabajar de forma autónoma & 5,96 \\
\hline Habilidades de investigación & 5,91 \\
\hline Toma de decisiones & 5,85 \\
\hline Liderazgo & 5,52 \\
\hline Planificación y gestión del tiempo & 5,21 \\
\hline Comunicación escrita & 5,11 \\
\hline & \\
\hline Opiniones personales & \\
\hline ¿Crees que la realización del trabajo en grupo ha sido positivo respecto a?: \\
Puntúa de 0-10 (0 muy en desacuerdo 10 totalmente de acuerdo) las siguientes afirmaciones: \\
\hline Ha ayudado a ver la aplicación práctica de la asignatura & 6,50 \\
\hline Permite relacionar todos los temas de la asignatura & 6,32 \\
\hline Requiere más tiempo del previsto por el equipo docente & 6,3 \\
\hline Realizando el trabajo he aprendido cosas útiles & 6,1 \\
\hline Me ha ayudado a entender mejor la asignatura & 5,46 \\
\hline Me ha servido para preparar el examen final de la asignatura & 3,93 \\
\hline El esfuerzo se corresponde con la valoración en la nota final & 3,45 \\
\hline Preferiría realizar el trabajo de forma individual & 2,50 \\
\hline Contesta (0 si y l no) las siguientes afirmaciones/preguntas & 0,29 \\
\hline aVolverías a repetir el trabajo? & 0,25 \\
\hline Globalmente estoy satisfecho con la realización del trabajo & \\
\hline
\end{tabular}

Es decir, hay alumnos que han aprobado la asignatura aprobando el examen final a pesar de no haber realizado todas las actividades de evaluación continua. Pero por otro lado, debemos analizar si el método de evaluación acumulativa por actividades lleva a que los alumnos aprueben en mayor medida la asignatura aún siendo incapaces de superar el examen final, ya que hay 29 alumnos que superan la asignatura pero no el examen.

En primer lugar, analizamos la diferencia de medias en las actividades de evaluación continua entre los alumnos que aprueban la asignatura pero no el examen y los que aprueban el examen y la asignatura. En este análisis se aprecia que los alumnos que aprueban la asignatura no aprobando el examen tienen una menor nota en todas las pruebas parciales, no es significativa al $5 \%$ la diferencia alcanzada en la nota de participación en clase y no resulta significativa al 10\% la alcanzada en el trabajo en grupos. En segundo lugar, analizamos la diferencia de medias entre los alumnos que 
aprueban la asignatura pero no el examen y los que no aprueban ni examen ni la asignatura. Los resultados obtenidos muestran como los alumnos que aprueban la asignatura no aprobando el examen no sólo es porque tengan una mayor nota media en todas las pruebas parciales sino que también alcanzan mayor puntuación en la realización del trabajo y en la participación interactiva en clase, siendo dichas diferencias significativas en todos los casos al $1 \%$.

Los anteriores resultados nos llevan a analizar en última instancia, si la realización del trabajo en grupo y la participación en clase, que parece que no influyen positivamente sobre la probabilidad de aprobar el examen, tienen una influencia positiva sobre la probabilidad de superar la asignatura.Dado que estas actividades forman parte de la nota evaluación continua (30\% sobre la nota final) se espera un efecto positivo, a pesar de que para aprobar la asignatura se deba alcanzar un mínimo de un $40 \%$ en la calificación del examen final ( $70 \%$ sobre la nota final). Además, recuérdese que las ponderaciones de las distintas pruebas suponían cada una un $5 \%$ en la nota final, la participación un $7 \%$ y el trabajo un $8 \%$ por lo que a priori podría esperarse que la influencia de dichas variables fuera proporcional a su peso en la calificación final.

La Tabla 4 recoge las estimaciones probit y logit de la relación entre la probabilidad de aprobar la asignatura y las variables independientes mencionadas y los $\mathrm{p}$ - valores asociados a los coeficientes. También se muestran los efectos marginales (E.M.) de los cambios en las variables independientes sobre el valor de la variable dependiente observada. Los efectos marginales han sido calculados nuevamente para cada observación de la muestra, obteniendo los efectos medios como promedio de los efectos individuales.

TABLA4. ESTIMACIONES DE LOS MODELOS PROBIT Y LOGIT PARA LA

PROBABILIDAD DE APROBAR LA ASIGNATURA

\begin{tabular}{|c|c|c|c|c|}
\hline \multicolumn{5}{|c|}{ PROBABILIDAD DE APROBAR LA ASIGNATURA } \\
\hline & \multicolumn{4}{|c|}{$\begin{array}{l}\text { Variable dependiente: Aprueba la asignatura }=1 \\
\text { No aprueba la asignatura=0 }\end{array}$} \\
\hline & \multicolumn{2}{|c|}{$\begin{array}{l}\text { Modelol } \\
\text { Probit }\end{array}$} & \multicolumn{2}{|c|}{$\begin{array}{l}\text { Modelo } 2 \\
\text { Logit }\end{array}$} \\
\hline & Coef. & E.M & Coef. & E.M \\
\hline Prueba 1 & $0,14\left(^{*}\right)$ & 0,02 & $0,27(* *)$ & 0,03 \\
\hline Prueba 2 & $0,17^{(* * *)}$ & 0,03 & $0,29\left(^{* * *}\right)$ & 0,03 \\
\hline Prueba 3 & $0,19^{(* * *)}$ & 0,03 & $0,33\left(^{* * *}\right)$ & 0,03 \\
\hline Trabajos & $0,73\left(^{*}\right)$ & 0,11 & $1,18\left(^{*}\right)$ & 0,12 \\
\hline Participación & $0,18(* *)$ & 0,03 & $0,33(* *)$ & 0,03 \\
\hline Constante & $-10,80\left({ }^{* * *}\right)$ & & $-18,20\left({ }^{* * *}\right)$ & \\
\hline Log likelihood & $-49,57$ & & $-49,77$ & \\
\hline $\mathrm{AIC}$ & 0,75 & & 0,75 & \\
\hline SBC & 0,87 & & 0,87 & \\
\hline HQC & 0,80 & & 0,80 & \\
\hline $\mathrm{R}^{2}$ Mcfadden & 0,52 & & 0,52 & \\
\hline Hosmer-Lemeshow $X^{2}$ & 13,15 & & 10,65 & \\
\hline
\end{tabular}

El modelo 1 es un modelo probit y el modelo 2 es un modelo logit. Se aprecia en ambos modelos como las variables notas en las tres pruebas parciales tienen una influencia positiva sobre la posibilidad de aprobar la asignatura en todos los modelos, resultando significativas al $1 \%$ las pruebas 2 y 3 , y al $5 \%$ o $10 \%$ la prueba 1 . La menor significatividad de la primera prueba puede ser debido a que ésta se realiza al haber abordado los temas 1 a 4 , en los que repasa en gran medida los contenidos de la asignatura de Introducción a la contabilidad que se imparte en primer curso y se añaden otros nuevos relacionados, pero no se imparte realmente materia nueva 
como se realiza a partir del tema 5 (pruebas 2 y 3). La variable participación resulta significativa al $5 \%$ en los dos modelos, teniendo una influencia positiva con un efecto marginal tan elevado como la nota alcanzada en las distintas pruebas. Finalmente, y contrario a los modelos establecidos para la probabilidad de aprobar el examen final pero si coherente con nuestra hipótesis de partida inicial, la variable trabajo en grupo tiene una influencia positivas sobre la probabilidad de aprobar la asignaturas siendo significativa en los dos modelos al $10 \%$. Además, el valor del efecto marginal de esta variable indica que es el factor que tiene mayor peso sobre la probabilidad de aprobar la asignatura. Este resultado se mantendría aún teniendo en consideración la distinta ponderación que tienen cada una de las actividades realizadas en la nota final. En base a los p-valores asociados a la prueba de Hosmer-Lemeshow para los dos modelos analizados resulta que el test no es significativo, lo que implica un buen ajuste de los modelos.

Muchos de los alumnos que han superado la asignatura en Grado la hubiesen superado con un sistema de evaluación basado únicamente en el examen final como el aplicado en Licenciatura. Sin embargo, un número destacable de alumnos han superado la asignatura gracias a la elaboración del trabajo en grupo, la participación activa en el aula y la superación de las distintas pruebas parciales. También existen dos alumnos que son incapaces de superar la asignatura aún superando el examen final dada la baja o nula puntuación en las actividades de evaluación continua. Esto lleva a plantearse la conveniencia o no de modificar el sistema actual de evaluación. Una posible solución a la problemática expuesta sería que el alumno, en función de su preferencia pudiera elegir entre un examen final del curso y una evaluación acumulada por actividades. Dado que el alumnado de la asignatura es muy heterogéneo (alumno en primera matrícula, repetidores grado, cambio desde licenciatura en $A D E$, etc.) resulta factible pensar que dicho alumnado puede tener diversa motivación y modo de estudio por lo que preferirían distintos sistemas de evaluación. Ello nos ha llevado a realizar en la encuesta anónima a los alumnos que han realizado el trabajo en grupo, preguntas acerca de la satisfacción con el sistema de evaluación de la asignatura (Tabla 5).

TABLA 5: ENCUESTA DE SATISFACCIÓN CON El SISTEMA DE EVALUACIÓN

\begin{tabular}{|l|c|}
\hline Preferiría que la evaluación consistiese básicamente en: & $9,1 \%$ \\
\hline El sistema actual en las convocatorias de Junio y Julio & $4,0 \%$ \\
\hline Un único examen final (nota sobre 10 puntos) en las convocatorias de Junio y Julio & $18,3 \%$ \\
\hline $\begin{array}{l}\text { El método actual en la convocatoria de Junio y un examen final (sobre 10 puntos) en } \\
\text { Julio }\end{array}$ & \\
\hline $\begin{array}{l}\text { Dos sistemas alternativos: un único examen final (nota sobre 10 puntos) para aquellos } \\
\text { alumnos que no deseen hacer la evaluación continua y el sistema actual basado en } \\
\text { evaluación continua y examen final. }\end{array}$ & $61,5 \%$ \\
\hline $\begin{array}{l}\text { Establecer el sistema de evaluación basado en un único examen final (nota sobre } 10 \\
\text { puntos) para aquellos alumnos repetidores y el sistema actual basado en evaluación } \\
\text { continua y examen final para el resto (alumnos en primera convocatoria). }\end{array}$ & $7,1 \%$ \\
\hline
\end{tabular}

Tanto el sistema actual implantado en la asignatura como el que resultaría del sistema tradicional son los menos preferidos por el alumnado, con un $9,1 \%$ y $4 \%$ de los votos. La opción más preferida por los alumnos es la de permitir la posibilidad de que el 
alumnado decida entre realizar el sistema tradicional y el nuevo sistema de evaluación (61,5\% de los votos). El resto de los alumnos preferiría que se hicieran distinciones en función de si el alumno es o no repetidor $(7,1 \%)$ o de la convocatoria en la que se presentase a examen (18,3\%). Pese a las anteriores preferencias, una mayoría (52\%) de los encuestados se mostraron satisfechos globalmente con el sistema de evaluación empleado en la asignatura ${ }^{5}$. Por tanto y, como es lógico, parece que la mayoría de los alumnos prefieren contar con diferentes posibilidades de evaluación para así escoger aquella que consideren más oportuna en función de sus necesidades puntuales.

En última estancia pretendemos analizar la evolución del rendimiento obtenido en el grado frente al sistema tradicional de licenciatura. Molero (2007) no encuentra diferencias significativas a nivel estadístico entre las calificaciones del alumnado del sistema tradicional y las obtenidas por una muestra reducida de participantes en una experiencia piloto de implantación del EEES. En la Tabla 6 podemos observar la evolución temporal de distintos indicadores del rendimiento académico del alumnado en el grado y en la licenciaturab.

TABLA 6: EVOLUCIÓN TEMPORAL DEL RENDIMIENTO ACADÉMICO

\begin{tabular}{|c|c|c|c|c|c|}
\hline Curso & $\mathbf{2 0 1 1 / 2 0 1 2}$ & $\mathbf{2 0 1 0 / 2 0 1 1}$ & $\mathbf{2 0 0 9 / 2 0 1 0}$ & $\mathbf{2 0 0 8 / 0 9}$ & $\mathbf{2 0 0 7 / 2 0 0 8}$ \\
\hline Titulación & Grado & Grado & Licenciatura & Licenciatura & Licenciatura \\
\hline $\begin{array}{c}\text { Tasa aprobados sobre } \\
\text { matriculados }\end{array}$ & $26,46 \%$ & $44,00 \%$ & $23,03 \%$ & $29,17 \%$ & $20,99 \%$ \\
\hline $\begin{array}{c}\text { Tasa presentados } \\
\text { media }\end{array}$ & $62,13 \%$ & $67,89 \%$ & $35,08 \%$ & $35,96 \%$ & $37,24 \%$ \\
\hline $\begin{array}{c}\text { Nota promedio } \\
\text { alumnos aprobados }\end{array}$ & 1,55 & 1,31 & 1,23 & 1,37 & 1,29 \\
\hline
\end{tabular}

Notas: La tasa de aprobados ha sido calculada como el número de alumnos que superan la materia sobre el número total de alumnos matriculados en cada curso. La tasa de presentados media ha sido calculada como la media aritmética de la tasa de presentados a examen en cada año académico, entendido como oportunidad de examen en grado (Junio o Julio) o convocatoria en Licenciatura (Junio o Septiembre).

En Grado la tasa media de presentados a examen es muy superior a la experimentada en Licenciatura, un $65,01 \%$ frente a un $36,09 \%$ respectivamente. En lo relativo a la tasa de aprobados sobre el total de alumnos matriculados en la materia, después del incremento que se dio en el curso 2010/11 como resultado de que para la amplia

\footnotetext{
${ }^{5}$ En este trabajo no hemos analizado los factores que influencian la probabilidad de valorar satisfactoriamente el sistema de evaluación. Florido et al. (2011) encuentran que las mujeres tienen una mayor probabilidad de valorar negativamente el sistema de evaluación continua en el marco el EEES, pero que es independiente de las notas alcanzadas por los alumnos.

${ }^{6}$ A la hora de comparar el grado y la licenciatura debemos advertir el carácter anual de la asignatura de licenciatura cuyo programa se ve en grado dividido en dos asignaturas: la que nos ocupa de segundo curso de 6 ECTS y otra de tercer curso de 4,5 ECTS. Además como ajuste a los créditos ECTS la carga de la asignatura es menor por lo que se han reajustado a la baja los contenidos. Destaca el menor número de alumnos matriculados en grado respecto a licenciatura debido fundamentalmente a dos factores. Primero, a que se han reducido los alumnos de nuevo ingreso en la facultad. Segundo, al elevado número de repetidores que históricamente tiene esta asignatura en licenciatura y que todavía no se han cambiado totalmente a Grado, ya que la USC les ha otorgado hasta el año 2011/12 la posibilidad de superar esta asignatura sin derecho a docencia.
} 
mayoría de los alumnos era la primera convocatoria de la asignatura, la tasa cae en el curso $2011 / 12(26,46 \%)$ a niveles ligeramente superiores a la media de la licenciatura $(24,40 \%)$. Finalmente, destacar que las notas promedio de los alumnos se establecen en una media de 1,43 en grado frente al 1,30 de licenciatura, calculadas como la media ponderada de las notas categorizadas de los alumnos que superan la asignatura (aprobado=1, notable $=2$; sobresaliente $=3$; matrícula de honor $=4$ ). Además, a diferencia de los resultados obtenidos por Pascual et al. (2011) no encontramos que el sistema de evaluación acumulativa por actividades empeore la calificación final de la asignatura de aquellos alumnos que sacan mejores notas en el examen final, sino que, en general, la mejora.

\section{CONCLUSIONES}

El nuevo sistema de evaluación impuesto en la asignatura de Contabilidad Financiera I en el Grado en ADE de la USC es diferente del sistema tradicional de Licenciatura, en el cual la evaluación era realizada únicamente en base a la nota alcanzada en el examen final. En este sentido una diferencia formal es que en el nuevo sistema se realiza una evaluación acumulativa por actividades, en las que se busca una mejora del aprendizaje del alumnado debido a su interacción en las aulas y en la resolución colaborativa y cooperativa de los trabajos presentados por el profesor. Estas actividades además conllevan el desarrollo de habilidades no sólo específicas del conocimiento contable, sino también intelectuales, personales, interpersonales y de organización. Un número importante de alumnos (64) que han aprobado la asignatura con el sistema actual, hubieran obtenido el mismo resultado con el sistema tradicional dado que ha superado el examen final. Sin embargo, es destacable que numerosos alumnos (29) han sido capaces de aprobar la asignatura aún no aprobando el examen final, aunque sí que alcanzaron un mínimo del $40 \%$ en la calificación de cada una de sus partes (test y ejercicios). Estos datos muestran que mediante el nuevo sistema de evaluación se amplía la posibilidad de alcanzar un aprobado por parte del alumno, de forma que el mismo puede mostrar su trabajo a lo largo del curso y no exclusivamente en un momento puntual. Por tanto, parece que esto cumple con uno de los objetivos de los sistemas de evaluación continua.

Los alumnos que aprueban el examen final tienen, en media, una mayor nota en cada una de las actividades de evaluación continua que los alumnos que no lo superan. De esta forma, las notas alcanzadas en todas las actividades de evaluación continua correlacionan significativa y positivamente con la probabilidad de aprobar el examen final. Sin embargo, cuando se estiman modelos logit y probit binomiales que estudian como variable independiente la probabilidad de aprobar el examen final y como variables dependientes las puntuaciones obtenidas en las actividades desarrolladas a lo largo del cuatrimestre destacan dos hechos. En primer lugar, las notas alcanzadas en las pruebas parciales tienen una influencia positiva y significativa sobre la probabilidad de aprobar el examen final. En segundo lugar y contrario a nuestras hipótesis de partida iniciales, las variables dependientes que indican la puntuación obtenida por los alumnos en la participación activa en el aula y en los trabajos en grupo no tienen influencia positiva sobre la probabilidad de aprobar el examen final. Si bien este hecho podría estar afectado por el reducido peso de las variables "participación" (7\%) y "trabajo en grupo" (8\%) para superar la asignatura con relación al otorgado al examen final (70\%). Por tanto, parece que las pruebas previas, al estar basadas en contenidos similares a los del examen final, permiten al alumno adquirir las competencias necesarias para superar el mismo. Por su parte, el trabajo en grupo facilita en mayor grado que los alumnos adquieran otro tipo de capacidades, como el trabajo en equipo, o que vean la utilidad de los contenidos. Estas habilidades si bien 
son necesarias para el desarrollo del alumno pueden tener un menor efecto directo sobre las posibilidades de aprobar el examen final.

Los alumnos que aprueban la asignatura pero no el examen final tienen en media una mayor nota en cada una de las actividades de evaluación continua que los alumnos que no aprueban la asignatura. Sin embargo, cuando comparamos las notas medias entre los alumnos que superando la asignatura aprueban o no el examen final encontramos que no existen diferencias entre las notas alcanzadas en la participación activa en el aula y en la realización del trabajo en grupo. Nuevamente estimamos modelos logit y probit cuya variable independiente es la probabilidad de aprobar la asignatura y como variables dependientes las puntuaciones obtenidas en las actividades de evaluación continúa. En estos modelos las notas alcanzadas en las pruebas parciales, la participación activa en el aula y en los trabajos en grupo tienen influencia positiva y significativa sobre la probabilidad de aprobar la asignatura. Además, el mayor efecto marginal se corresponde con la nota del trabajo en grupo. La interpretación de estos resultados nos lleva a que los alumnos que superan esta materia tienen competencias en todas las actividades que forman el sistema de evaluación planteado. Por tanto, podemos señalar que todas ellas son útiles y tienen una cierta complementariedad. En especial destaca la influencia del trabajo en grupo, dicho trabajo parece que desarrolla las capacidades o competencias necesarias para un profesional de la actividad contable, en base a los resultados obtenidos de una encuesta a los alumnos.

Analizando el sistema de evaluación actual empleado en la asignatura encontramos que una mayoría de los alumnos se muestran globalmente satisfechos, aunque preferirían elegir la posibilidad de ser evaluados en base al sistema tradicional o al nuevo sistema de evaluación. Esto puede ser debido a que la adaptación al nuevo sistema implica un trabajo autónomo superior del alumno y necesita del estudio continuo de la asignatura, hecho que a muchos alumnos les puede parecer una carga de trabajo excesiva. Además del análisis comparativo del rendimiento obtenido a través de distintos indicadores se observa un aumento de los mismos en el grado. La razón reside en que los alumnos mejoran la calificación obtenida en el examen final gracias a las actividades de evaluación continua.

Por último, debemos indicar que los resultados obtenidos en este trabajo pueden ser de gran utilidad en aquellas universidades y/o materias que tengan sistemas de evaluación similares a los aquí presentados, donde el examen tienen un peso elevado en la nota final. Como limitaciones del trabajo realizado hemos de mencionar que no se han tenido en cuenta variables de control a veces empleadas en la literatura, tales como el género de los alumnos o su rendimiento académico previo. Además, hay que señalar que la nota del trabajo en grupo puede no resultar en algunos casos indicativa del trabajo o aprendizaje realizado por los alumnos debido al gran número de trabajos presentados (119) y la alta dificultad percibida por el profesorado para conocer las tareas efectivamente realizadas por cada uno miembro de un mismo grupo. Esto nos ha llevado a considerar en próximos cursos completar la valoración del mismo añadiendo al proceso una evaluación individual mediante un cuestionario.

\section{BIBLIOGRAFÍA}

AKAIKE, H. [1973]: "Information Theory and an Extension of the Maximum Likelihood Principle", en N. Petrov y F. Csake (editores) The Second International Symposiumon Information Theory, pp.267-281. Hungary: Akademiai Kiado.

ALDERSON, J. C., Y WALL, D. [1993]: "Does wash back exist?", Applied Linguistics, n 14, pp. $115-129$. 
ARUlampalam, W., NAYlor, R. y SMith, J. [2012]: "Am I Missing Something? The Effects of Absence from Class on Student Performance", Economics of Education Review, Vol. 31, n. 4, pp. 363-375.

BARKLEY, A. [2006]: "The Determinants of College Student Performance: The Role of Assessment Method", WAEA anual meetings, Alaska (EE.UU.), Junio, pp. 2830.Disponible en:http://waeaonline.uwagec.org/2006AssnMtg/Proceedings/Barkley_IIID.pdf

DELANEY, L.; HARMON, C. y RYAN, M. [2011]: "Does Lecture Attendance Matter for Grades? Evidence from Longitudinal Tracking of Irish Students", Working paper, disponible en http://ciisn.files.wordpress.com/2011/01/martin-ryan-_-doeslecture-attendance-matter-for-grades_working-paper.pdf

DEVAdOSS, S. y FOLTZ, J. [1996]: "Evaluation of factors influencing student class attendance and performance ", American Journal of Agricultural Economics, $\mathrm{n}^{\circ}$ 78, pp. 499-507.

DuRDEN, G. C. y ElLIS, L. V. [1995]: "The effect of attendance on student learning in Principles of Economics", American Economic Review, n85, pp. 343-346.

Florido DE LA NuEZ, C., JimÉNEZ GONZÁleZ, J.L. y SANTANA MARTíN, I. [2011]: "Obstáculos en el camino hacia Bolonia. Efectos de la implantación del EEES sobre los resultados académicos"; Revista de Educación, n³54, pp. 629-656.

FREDERIKSEN, N. [1984]: "The real test bias. Influences of testing on teaching and learning", American Psychologist, $\mathrm{n}^{\circ}$ 39, pp. 193-202.

HANNAN, E.J. Y QUINN, B.G. [1979]: "The determination of the order of an autoregression", Journal of the Royal Statistical Society B, 41, pp. 190-195.

HOSMER, D. W. Y LeMESHOW, S. [1989]: Applied Logistic Regression. New York: John Wiley\&Sons, Inc.

LÓPEZ-PASTOR, V.M. [2010]:"La evaluación por competencias en la docencia universitaria: posibilidades de la evaluación formativa y compartida" en Marín, M. et al.: Actas VI Intercampus. Evaluación de las competencias en los nuevos grados. Cuenca: UCLM, pp.40-53.

MARTINS, P. Y WALKER, I. [2006]: "Student Achievement and University Classes: Effects of Attendance, Size, Peers, and Teachers", IZA Discussion Papers $n^{\circ} 2490$, Institute for the Study of Labor (IZA).

MCFADDEN, D. [1974]:"Conditional logit analysis of qualitative choice behavior," en Frontiers inEconometrics, Zarembka, P. (ed.) Academic Press,New York, pp. 105-142.

MEDINA, R. [2005]: "Misiones y funciones de la universidad en el espacio europeo de educación superior", Revista Española de Pedagogía, nº. 230, pp. 17-42.

MOLERO, D. [2007]: "Rendimiento académico y opinión sobre la docencia del alumnado participante en experiencias piloto de implantación del Espacio Europeo de Educación Superior", RELIEVE, Vol. 13, n. 2, pp. 175-190.

Pascual, D., CAMACho, M., URquía, E. y Müller, A. [2011]: "¿Son los nuevos criterios de evaluación en el marco del EEES adecuados para valorar el rendimiento académico de los alumnos?. Experiencia en Contabilidad Financiera", Revista Educade, $n^{\circ} 2$, pp. $67-83$.

Romer, D. [1993]: "Do Students Go to Class? Should They?",The Journal of Economic Perspectives, Vol. 7, n. 3, pp. 167-74.

RODGERS, J.R. [2002]: 'Encouraging tutorial attendance at university did not improve performance', Australian Economic Papers, September, pp. 255-266.

RúA A. y GONZÁLEZ, C. I. [2004]: "Predicción del rendimiento académico final a partir de pruebas previas en asignaturas cuantitativas", XII Jornadas ASEPUMA. Murcia (Spain). Disponible

en: http://www.uv.es/asepuma/XII/comunica/rua_gonzalez.pdf

SCHWARz, G. [1978]: "Estimating the dimension of a Model", The Annals of Statistics, 6, pp. 461-464. 
StANCA, L. [2006]: "The effects of Attendance on Academic Performance: Panel Data Evidence for Introductory Microeconomics", The Journal of Economic Education, $\mathrm{n}^{\circ}$ 37, pp. 251-266.

TEJEDOR, F. J. [2003]: "Poder explicativo de algunos determinantes del rendimiento en los estudios universitarios", Revista española de Pedagogía, No. 224, pp. 5-32.

TEJedOR, F. J. y GARCÍA-VALCÁRCEL, A. [2007]: "Causas del bajo rendimiento del estudiante universitario (en opinión de profesores y alumnos). Propuestas de mejora en el marco del EEES", Revista de Educación, n. 342, pp. 443-473.

VILLARDÓN, L. Y YÁNIZ, C. [2004]: "El aprendizaje cooperativo y el nuevo concepto de evaluación", IV Jornada de Aprendizaje Cooperativo, UPC, Barcelona. 